\title{
EFECTOS GÉNICOS Y HETEROSIS EN POBLACIONES PARENTALES Y POBLACIONES DERIVADAS DE MAÍZ (Zea mays L)*
}

\author{
Enrique Navarro G. y Fernando Borrego E.**
}

\begin{abstract}
RESUMEN
En el presente estudio se utilizó el modelo GardnerEberhart (1966) y se involucró a 8 poblaciones parentales de maíz y sus poblaciones derivadas. El objetivo fue estimar los efectos génicos acumulativos (aditivos, dominantes y heteróticos), con la finalidad de justificar un programa de hibridación. Para rendimiento de grano, dominancia intravarietal (dj) explicó 55,54\% de las medias generacionales, sugiriendo suficiente variabilidad genética dentro de las poblaciones. Efectos heteróticos contribuyeron con 12,11\% indicando la poca diferencia que existe en la frecuencia génica para rendimiento de grano. Altura de planta siguió el mismo patrón que rendimiento, ya que los efectos dominantes fueron los más importantes. Sin embargo, los loci homocigotes (aj) fueron importantes en explicar variabilidad gen ética en días a floración y no. de mazorcas. Para rendimiento de grano, la cruza Pob. 32 x Pob. 21 fue la que maximizó los efectos heteróticos. La población 21 exhibió mayor heterosis en promedio, de tal suerte que en combinación con Pob. 32 y/o $\mathrm{CN}(\mathrm{S})-\mathrm{C}_{3}$, entre otras, podría ser material promisorio para un programa de selección recíproca recurrente.
\end{abstract}

\begin{abstract}
Genic effects and heterosis of parental populations and related corn (Zea mays L.) populations. This study with the Gardner-Eberhart model (1966) involved eight maize parental populations and their related populations. The objectives were to estimate the cumulative gene effects (additive, dominance and heterotic) which could The justify support of a maize hybrid programo The grain yield inter-varietal dominance effects accounted for $55.54 \%$ of the generation means, suggesting large genetic variability within populations. Heterotic effects accounted for $12.11 \%$, indicating little difference in gene frequency for loci controlling grain yield. Plant height followed the same pattero as grain yield, since dominance effects were the most important. However, homozygote loci (aj) were important in explaining genetic variability for days to bloom and number of ears. For grain yield, the cross Pop. 32 x Pop. 21 maximized the heterotic effects. Population 21 exhibited a high average heterosis, so we suspect that its, combination with Popo 32 and $\mathrm{CN}(\mathrm{S})-\mathrm{C}_{3}$, among others, would be a great genetic material for a Reciprocal Recurrent Selection Programo
\end{abstract}

\section{INTRODUCCIÓN}

El éxito de cualquier programa genético con énfasis en el desarrollo de líneas endocriadas para la formación de híbridos, dependerá de la elección del germoplasma base a considerarse dentro del programa de mejoramiento. Asímismo, el mejorador requiere de un conocimiento amplio del tipo de acción génica de los materiales en estudio de los caracteres de mayor importancia económica tales como alto potencial de rendimiento, resistencia genética a las mayores plagas y enfermedades dentro de la población de macroambientes del área bajo estudio. Lo anterior permitirá elegir el mejor esquema de mejoramiento que maximize la varianza genética y de esta forma incrementar y fijar la frecuencia de genes favorables en la población parental. De tal forma que elección de variedades para ser usados como población base en un programa de selección recurrente dependerá de, 1) media de comportamiento de los variedades, 2) heterosis varietal, y 3) de la variación genética dentro de la población.

El diseño de cruzamiento dialélico ha sido usado más que cualquier otro tipo de diseño de apareamiento para evaluar el potencial genético entre poblaciones de maíz. La teoría para la estimación de varianza genética y efectos genéticos usando este tipo de diseños fueron propuestos por Hayman (1954, 1958 y 1960), y Griffing (1956). Sin embargo, Gardner-Eberhart (1966) recomendaron el uso de cruzas varietales en un dialélico para un mejor entendimiento de las cruzas dialélicas entre variedades de polinización libre de maíz. Un gran número de

\footnotetext{
* Trabajo presentado en la XXXVIII Reunión Anual del PCCMCA celebrada en Managua, Nicaragua, 23-27 de marzo de 1992.

** Maestros Investigadores del Departamento de Fitomejoramiento. Universidad Aut. Agr. Antonio Narro, (UAAAN) Saltillo, Coah México 25315.
} 
investigadores han hecho uso del modelo GardnerEberhart (1966) para detectar material promisorio en cultivos como maíz. Castro et al. (1968) evaluaron cinco poblaciones de maíz y 35 poblaciones derivadas de ellas. Para rendimiento, el $52,4 \%$ de la variación total fue explicado por dominancia intravarietal, los efectos aditivos fueron significativos pero de menor magnitud. Heterosis o efecto de dominancia intervarietal aunque significativos, contibuyeron con tan sólo $6,5 \%$, dejando una pequeña porción sin explicar $(1,8 \%)$. Lo anterior indica que existe suficiente variabilidad genética dentro de poblaciones, y que poca diferencia en frecuencias génicas existe entre las poblaciones para rendimiento. Cortez et al. (1985) realizaron cruzas dialélicas en poblaciones tropicales de maíz desarrolladas por CIMMYT. Los autores reportan que los efectos de dominancia intravarietal fueron los que mayormente contribuyeron en la expresión de rendimiento de grano, sugiriendo adecuada variabilidad genética dentro de las poblaciones. Efectos de dominancia fueron también importantes en la expresión de porciento de polen, días a floración, altura de planta y mazorca. Efectos aditivos fueron significativos $(\mathrm{P} \leq 0,01)$ para todos los caracteres pero de menor magnitud. Los patrones heteróticos observados, sugieren que hay buen nivel de divergencia genética en alguna de las poblaciones. Estudios recientes conducidos por Crossa et al. (1990) indican que para rendimiento de grano y días a floración, los efectos de variedades ( $\mathrm{Vj}$ ) y heterosis (hjj') contribuyeron significativamente. Efectos genéticos no aditivos, fueron mas importantes en controlar rendimiento (50\%) en relación a días a floración (31\%). Para rendimiento, la cruza que maximizó la heterosis específica fue la población 36 x población 24 , indicando que podría ser una buena elección para iniciar un programa de selección recíproca recurrente. Con la finalidad de determinar la habilidad combinatoria y los patrones heteróticos en germoplasma de maíz generadas por el CIMMYT, Beck et al. (1990) usaron el Análisis III de Gardner-Eberhart (1966). Ellos encontraron que la heterosis promedio fue altamente significativa para rendimiento de grano, altura de planta y mazorca. La comparación padres vs. cruzas explicada por la heterosis promedio representada por un set en particular de padres, es atribuida a efectos genéticos no aditivos. Sin embargo, el 91,5\% de la suma de cuadrados totales debido a cruzas, es explicado por ACG, mientras que sólo el 8,5\% es atribuida a la ACE, indicando la importancia de los efectos gen éticos aditivos en relación a los efectos no aditivos para el carácter arriba mencionado.
Tendencia similar fue observada para el resto de los caracteres tales como días a floración, altura de planta y mazorca. También observaron que para rendimiento, la heterosis sobre el mejor padre fue baja para la mayoría de las cruzas, sin embargo, niveles moderados fueron observados en la población 49 x población $26(9,6 \%)$.

Los objetivos de presente trabajo fueron:

a) Obtener información de los efectos aditivos, de dominancia así como de los efectos heteróticos entre poblaciones desarrollados por la UAAAN y el Programa de maíz del CIMMYT y

b) Determinar patrones heteróticos y cuantificar la magnitud de los mismos y así justificar la producción de híbridos.

\section{MATERIALES Y METODOS}

Para la realización de este estudio se utilizaron las poblaciones VS-201 y Compuesto Norteño $(\mathrm{CN})$, sus tres ciclos de selección recurrente a través de líneas $\mathrm{S}_{1}$ per se (S) y líneas $\mathrm{S}_{1}$ por cruza de prueba (CP), poblaciones 32 y 21 del programa de maíz del CIMMYT, y sus poblaciones derivadas. Estas se obtuvieron en la primvera de 1988 en Buenavista, Coahuila donde las ocho poblaciones se sembraron para generar las $F_{1}$ 's. Se sembraron 20 surcos de $10 \mathrm{~m}$ de longitud de cada entrada. En floración, se hicieron cruzamientos entre las 28 cruzas (directas y recíprocas). Polen de 50 plantas de una población y viceversa, obteniéndose 100 mazorcas de cada cruza poblacional. En diciembre de ese mismo año en Tepalcingo, Morelos se sembraron las 28 cruzas $\left(F_{j j}{ }^{\prime}\right)$ y los ocho progenitores. Las cruzas autofecundadas $\left.\left(\mathrm{F}_{\mathrm{jj}}\right)^{\prime}\right)$, se obtuvieron autofecundando 100 plantas por parcela. Las cruzas recombinadas $\left(\mathrm{F}_{\mathrm{jj}}{ }^{\prime}\right)$ se produjeron haciendo polinizaciones planta a planta, utilizando la espiga una sola vez, totalizando 100 mazorcas por parcela. Para obtener los progenitores autofecundados y recombinados, se sembraron dos surcos por progenitor. La mitad de ellos usados para autofecundar y el resto para generar los progenitores recombinados, siguiendo el mismo procedimiento anterior. En verano de 1989 en Buenavista, Coahuila, se sembraron las 100 entradas en un diseño de bloques al azar, agrupándose en cinco grupos dentro de cada una de las cuatro repeticiones. La unidad experimental consistió de dos surcos de 4,62 m de 22 plantas espaciados a $22 \mathrm{~cm}$, dando una densidad de 56818 plantas ha). Prácticas culturales fueron de acuerdo a los procedimientos mínimos estandard de la región. Las 
variables experimentales fueron: Días a flor, Altura de planta, Altura de mazorca, Mazorcas por cien plantas, y Rendimiento de grano en $\mathrm{t} / \mathrm{ha}^{-1}$ al $15 \%$ de humedad.

\section{Análisis Estadístico}

El modelo lineal aditivo de bloques al azar generó la variación de medias gene racionales y mediante la técnica de regresión múltiple fue posible la estimación de parámetros genéticos siguiendo el procedimiento de Gardner (1967) y Gardner y Eberhart (1966), para la estimación de efectos acumulativos de acuerdo al análisis 1.

\section{RESULTADOS Y DISCUSIÓN}

Resultados experimentales de efectos acumulativos son sumarizados en el Cuadro 1 donde se observa que los loci heterocigotes $\left(\mathrm{d}_{\mathrm{j}}\right)$, fueron significativos $(\mathrm{P} \leq 0,01)$, representando el $55,54 \%$ de la variación total debido a medias generacionales. Lo anterior sugiere que existe una gran variabilidad dentro de poblaciones.

Resultados similares fueron obtenidos por (Castro $e t$ al. 1968) quienes reportaron que dominancia contribuyó con $52,4 \%$ de la variación total entre medias generacionales para rendimiento. La heterosis $\left(\mathrm{h}_{\mathrm{jj}}{ }^{\prime}\right) \mathrm{o}$ dominancia intervarietal fue significativa $(\mathrm{P} \leq 0,05)$, indicando la presencia de dominancia y diferencia en las frecuencias génicas, así como habilidad combinatoria general entre las variedades; esto coincide con lo reportado por (Gardner y Paterniani, 1967). La heterosis promedio (h) fue significativa $(\mathrm{P} \leq 0,01)$, explicando $2,07 \%$ de la variación total, indicando que en promedio las cruzas excedieron el valor promedio de los padres. Heterosis varietal $(\mathrm{Hj})$ fue significativa $(\mathrm{P} \leq 0,05)$ contribuyendo con un $4,78 \%$ de medias generacionales.

La heterosis promedio es una función lineal de la diferencia entre la media de un padre en sus cruzas y el promedio de todas las cruzas y la diferencia de la media de un padre y el promedio de todos los padres (Gardner, 1967).

Desviaciones del modelo presentaron significancia $(\mathrm{P} \leq 0,01)$, atribuyéndose a efectos epistáticos como a $\mathrm{x}$ a, a $\mathrm{x} \mathrm{d}, \mathrm{d} \times \mathrm{d}$, indicando que epistasis es importante en cruzas entre variedades de diferente origen (Robinson y Cockerham, 1961). Para días a floración el 51,52\% fue explicado por efectos aditivos, resultados semejantes a los obtenidos por Crossa et al. 1990.
Cuadro 1. Efectos acumulativos, cuadrados medios, y desviaciones del modelo de los siguientes caracteres, en la localidad de Buenavista, Coah. México, 1989.

\begin{tabular}{|c|c|c|c|c|c|}
\hline \multirow[b]{2}{*}{ FV } & \multirow[b]{2}{*}{$\mathrm{gl}$} & \multicolumn{4}{|c|}{ c u a d r a dos Med ios } \\
\hline & & $\begin{array}{l}\text { Rend. } \\
t / h a\end{array}$ & $\begin{array}{l}\text { Días a } \\
\text { Flor }\end{array}$ & $\begin{array}{l}\text { Alt. Plta. } \\
(\mathrm{cm})\end{array}$ & $\begin{array}{l}\text { No. de } \\
\text { Mazorcas }\end{array}$ \\
\hline Reps & 3 & 14,36 & $321,54 *$ & $17793,21 * *$ & $8637,67 * *$ \\
\hline Trats & 99 & $19,32 * \star$ & $99,27 \star \star$ & $1155,63 * \star$ & $843,35 * *$ \\
\hline $\mathrm{Aj}$ & 7 & 2,74 & $723,35 * *$ & $999,93 *$ & $1798,50 * *$ \\
\hline $\mathrm{dj}$ & 8 & $132,78 * *$ & $85,07 * \star$ & $8339,32 * *$ & 928,23 \\
\hline$h j j^{\prime}$ & 28 & $8,27 *$ & $60,20 * \star$ & 413,77 & 571,53 \\
\hline h & 1 & $39,63 * *$ & 3,59 & 1017,49 & 1667,13 \\
\hline hj & 7 & $13,05 *$ & $153,88 *$ & 615,52 & 266,80 \\
\hline$S \mathrm{jj}^{\prime}$ & 20 & 5,03 & $30,24 * *$ & 312,98 & 588,41 \\
\hline Desv. & 56 & $10,71 * *$ & $42,83 * *$ & 519,77 & $847,74 *$ \\
\hline Error & 297 & 5,55 & 13,96 & 380,15 & 507,83 \\
\hline
\end{tabular}

En altura de planta los efectos de dominancia $(58,31 \%)$ fueron más importantes que los aditivos $(6,12 \%)$. Finalmente, los efectos aditivos sólo fueron significativos $(P \leq 0,01)$ para mazorcas por cien plantas, a excepción de desviaciones de regresión. Estos resultados sugieren que dominancia $\mathrm{y}$ heterosis no fueron importantes en controlar número de mazorcas. Para rendimiento, las cruzas Pob. 32 x Pob. 21 (66\%), Pob. 21 x CN (CP)- $\mathrm{C}_{3},(51,46 \%)$ entre otras, tuvieron los mayores efectos de heterosis (H), Cuadro 2. Se observó que en general valores altos de heterosis estuvieron asociados con promedios altos. La población 21 en promedio es quien está contribuyendo con mayor variabilidad genética; es importante señalar que las cruzas que maximizaron la heterosis específica $(\mathrm{Sjj}$ '), no siempre tuvieron valores altos de heterosis, lo cual es contrario a lo indicado por Crossa et al. 1990, donde valores altos de heterosis específica se tradujeron en una máxima expresión de heterosis para rendimiento. En días a floración la cruza Pob. $32 \times$ Pob. 21 tuvo el valor de heterosis más bajo $(-17,44 \%)$, sin embargo, estuvo entre las cruzas de porte más alto (datos no publicados). Por el contrario la cruza VS-201 (S)-C $\mathrm{C}_{3}$ x VS-201 $\mathrm{C}_{0}$ tuvo el valor de heterosis más bajo $(-12,14)$ con una heterosis específica de $\left(\mathrm{S}_{\mathrm{jj}}{ }^{\prime}=15,72\right)$. En general se observó que germoplasma del VS-201 $\mathrm{C}_{0}$, su versión mejorada, y versión mejorada del $\mathrm{CN}$, puede ser material promisorio para reducir altura de planta, según muestran valores de heterosis. Para número de mazorcas $\mathrm{x}$ 100 plantas, la cruza Pob. $32 \times \mathrm{CN}(\mathrm{S})-\mathrm{C}_{3}$ exhibió la máxima heterosis, $(\mathrm{H})(31,13 \%)$ la misma que tuvo la heterosis específica más alta $\left(\mathrm{S}_{\mathrm{jj}}{ }^{\prime}=18.89\right)$ aunque fue no significativa. Considerando los patrones heteróticos el mejor germoplasma para iniciar un programa de hibridación sería Pob.32 x Pob. 21 o bien utilizar las poblaciones mejoradas del VS-201 (CP) y/o del CN (S) en combinación con los mencionados anteriormente. 
Cuadro 2. Medias predichas de las cruzas $\left(\mathrm{Y}_{\mathrm{jj}}{ }^{\prime}\right)$, heterosis $(\mathrm{H})$ y heterosis específica $\left(\mathrm{S}_{\mathrm{jj}}{ }^{\prime}\right)$ sobresalientes entre ocho pobalciones de Maíz. Buenavista, Coah. México, 1989.

\begin{tabular}{|c|c|c|c|}
\hline Cruza & $Y_{j j}^{\prime}$ & H & $S_{j j}{ }^{\prime}$ \\
\hline $\begin{array}{l}\text { Rendimiento (t/ha) } \\
\text { Pob. } 32 \times \text { Pob. } 21 \\
\text { Pob. } 21 \times \mathrm{CN}(\mathrm{S})-\mathrm{C}_{3} \\
\text { Pob. } 21 \times \mathrm{CS} 201 \mathrm{CP})-\mathrm{C}_{3} \\
\text { VS } 201(\mathrm{~S})-\mathrm{C}_{3} \times \mathrm{CN}(\mathrm{CP})-\mathrm{C}_{3} \\
\text { Pob. } 21 \times \mathrm{CN}(\mathrm{CP})-\mathrm{C}_{3}\end{array}$ & $\begin{array}{r}10,81 \\
10,45 \\
10,22 \\
10,02 \\
9,15\end{array}$ & $\begin{array}{l}66,00 \\
46,28 \\
49,20 \\
41,32 \\
51,46\end{array}$ & $\begin{array}{l}0,95 \\
0,21 \\
0,52 \\
2,53^{*} \\
0,25\end{array}$ \\
\hline $\begin{array}{l}\text { Floración } \\
\text { Pob. } 21 \times \text { VS } 201-C_{0} \\
\text { Pob. } 21 \times C N(S)-C_{3} \\
\text { Pob. } 32 \times \text { Pob. } 21 \\
\text { Pob. } 21 \times \text { VS } 201(C P)-C_{3} \\
\text { Pob. } 21 \times \text { VS } 201(\mathrm{~S})-C_{3}\end{array}$ & $\begin{array}{l}74,73 \\
72,32 \\
71,84 \\
70,48 \\
70,13\end{array}$ & $\begin{array}{r}-6,22 \\
-8,65 \\
-17,44 \\
-10,89 \\
-11,68\end{array}$ & $\begin{array}{r}1,75 \\
-0,23 \\
4,99 * \\
-1,74 \\
-1,29\end{array}$ \\
\hline $\begin{array}{l}\text { Altura de Planta (CD) } \\
\text { CN (S)-C } C_{3} \times C N(C P)-C_{3} \\
\text { VS } 201(C P)-C_{3} \times C N(C P)-C_{3} \\
\text { VS } 201(C P)-C_{3} \times C N(S)-C_{3} \\
\text { VS } 201(S)-C_{3} \times \text { VS } 201(C P)-C_{3} \\
\text { VS } 201(S)-C_{3} \times \text { VS } 201-C_{0}\end{array}$ & $\begin{array}{l}153,04 \\
150,19 \\
148,33 \\
146,42 \\
140,12\end{array}$ & $\begin{array}{r}-3,65 \\
-2,71 \\
-6,20 \\
-2,72 \\
-12,14\end{array}$ & $\begin{array}{r}-1,93 \\
-5,37 \\
-5,93 \\
-3,80 \\
-15,72\end{array}$ \\
\hline $\begin{array}{l}\text { No. de Mazorcas } \\
\text { Pob. } 32 \times \mathrm{CN}(\mathrm{S})-\mathrm{C}_{3} \\
\text { Pob. } 32 \times \text { VS } 201-\mathrm{C}_{0} \\
\mathrm{CN}(\mathrm{S})-\mathrm{C}_{3} \times \mathrm{CN}(\mathrm{CP})-\mathrm{C}_{3} \\
\text { VS } 201(\mathrm{~S})-\mathrm{C}_{3} \times \text { VS } 201(\mathrm{CP})-\mathrm{C}_{3} \\
\mathrm{CN}(\mathrm{S})-\mathrm{C}_{3} \times \text { VS } 201-\mathrm{C}_{0}\end{array}$ & $\begin{array}{l}151,03 \\
132,88 \\
131,83 \\
123,36 \\
125,11\end{array}$ & $\begin{array}{l}31,73 \\
16,16 \\
17,56 \\
19,51 \\
20,16\end{array}$ & $\begin{array}{r}19,89 \\
10,69 \\
6,52 \\
2,97 \\
11,37\end{array}$ \\
\hline
\end{tabular}

* significativo al $5 \%$ de probabilidad.

$H=$ Heterosis expresada en por ciento de la cruza predicha.

$\mathrm{Y}_{\mathrm{Jj}}{ }^{\prime}$, con respecto a la media predicha parental.

$\mathrm{S}_{\mathrm{jj}}{ }^{\prime}=$ Heterosis específica de la cruza $\mathrm{j} \times \mathrm{j}$.

\section{CONCLUSIONES}

Los efectos de dominancia intravarietal $\left(\mathrm{d}_{\mathrm{j}}\right)$ fueron importantes en la expresión de rendimiento de grano, tomando en consideración su mayor contribución a la variación total de medias generacionales. Similar tendencia se observó en altura de planta, donde los efectos de dominancia fueron más importantes que los aditivos. Sin embargo, en días a flor y número de mazorcas los efectos aditivos fueron importantes en la expresión de estos caracteres. Para rendimiento, las cruzas que maximizaron los efectos heteróticos fueron Pob. 32 x Pob. 21 y Pob 21 x CN(CP)-C 3 , se recomienda utilizar germoplasma de Pob. 21 dado sus valores promedios mas altos en cruzas. Se sugiere utilizar a las poblaciones 32,21 y a las poblaciones mejoradas de VS 201 (CP) y del $\mathrm{CN}(\mathrm{S})$, para iniciar un programa de selección recíproca recurrente donde se espera que las líneas endocriadas que se deriven de ellas maximizen la heterosis cuando se crucen entre sí.

\section{LITERATURA CITADA}

BECK, D.L.; S.K. VASAL; J. CROSSA. 1990. Heterosis and combining ability of CIMMYT's Tropical early and intermediate maturity maize (Zea mays L.) germplasm. Maydica 35: 279-285.

CASTRO, G.M.; C.O. GARDNER; J.H. LONNQUIST. 1968.Cumulative effects and the nature of heterosis in maize crosses involving genetically diverse race. Crop. Sci. 8:97-101.

CORTEZ, H.M.; A.c. RODRIGUEZ; M.G. GUTIERREZ; J.I. DURON; R.c. GIRON; M.G. OYERVIDES. 1985. Evaluation of broad-base improved populations of maize (Zea mays L.). I. Cumulative gene effects and heterosis. Univ. Aut. Agraria "Antonio Narro" Res.Pub. p. 1-43. Buenavista, Saltillo, México.

CROSSA, J.; S.K. VASAL; D.L. BECK. 1990. Combining ability estimates of CIMMYT's tropical late yellow maize germplasm. Maydica. 35:273- 278.

GARDNER, C.O. 1967. Simplified methods for estimating constants and computing sums of squares for a diallel cross analysis. Fitotec. Latinoamer. 4:11-22.

GARDNER, C.O.; E. PATERNIANI. 1967. A genetic modelused to evaluate the breeding potential of openpollinated varieties of corno Ciencia E Cultura 19:95101.

GARDNER, C.O.; SA. EBERHART. 1966. Analysis and interpretation of variety cross dialell and related populations. Biometrics 22:439-452.

GRIFFING, B. 1956. Concept of general and specific combining ability in relation to diallel crossing systems. Australian J. Biol. Sci. 9:463-493.

HA YMAN, B.I. 1954. The theory and analysis of diallel crosses Genetics 39:789-809.

1958. The theory and analysis of diallel crosses II. Genetics 43:63-85.

1960. The theory and analysis of diallel crosses III. Genetics 45: 157-172.

ROBINSON, H.F.; C.C. COCKERHAM. 1961. Heterosis and inbreeding depression in populations involving two open pollinated varieties of maize. Crop. Sci.1:6871. 